\title{
Combien de co-récepteurs pour les virus de l'immunodéficience humaine ?
}

On sait depuis l'année dernière que certains récepteurs de chimiokines sont utilisés par les virus de l'immunodéficience humaine (VIH-1 et VIH-2) comme des «co-récepteurs» associés à CD4 (m/s $n^{\circ} 8-9$, vol. 12 , p. 975). Ce rôle a d'abord été mis en évidence pour CXCR4, un récepteur de chimiokines de type $\mathrm{CXC}^{*}$, initialement désigné fusin $\left(\mathrm{m} / \mathrm{s} n^{\circ} 10\right.$, vol. 12, p. 1185). Il est utilisé par les souches VIH-1 dites "T-tropic», capables de se répliquer dans des lymphocytes $\mathrm{CD}^{+}$et les lignées cellulaires T. Par la suite, plusieurs groupes ont montré qu'un récepteur de CC chimiokines, CCR5, était utilisé par la quasi-totalité des souches de VIH-1, qu'elles soient de type «macrophage (M)-tropic» ou T-tropic. Ces dernières peuvent donc utiliser deux co-récepteurs, CCR5 et CXCR4. Le rôle primordial de CCR5 dans le cycle naturel de VIH-1 semblait indiqué par la résistance à l'infection des sujets $\triangle C C R 5$, porteurs homozygotes de mutations inactivant ce récepteur $\left(\mathrm{m} / \mathrm{s} n^{\circ} 4\right.$, vol. $\left.13, p .613\right)$. Si ces données restent valables, plusieurs observations récentes montrent que CCR5 et CXCR4 ne sont pas les seuls corécepteurs possibles pour VIH-1, et surtout pour VIH-2 et les lentivirus simiens apparentés (SIV). Par ailleurs, le fait qu'une même souche

\footnotetext{
* Les chimiokines sont des protéines solubles de 60 à 80 acides aminés, ayant pour la plupart un rôle dans le chimiotactisme des leucocytes. On en connaît maintenant plus de vingt. Elles sont classées en sous-groupes définis par la position de deux cystéines conservées de leur domaine amino-terminal, soit adjacentes pour les chimiokines $C C$ (ou $\beta)$, soit séparées par un résidu pour les chimiokines CXC (ou $\alpha)$. On connaît également une chimiokine de type $C$, la lymphotactine, et une chimiokine de type $\mathrm{CX}_{2} C$ ancrée à la membrane, la neurotac-
}

puisse utiliser plusieurs co-récepteurs amène à discuter la nature et la spécificité de leur interaction avec l'enveloppe virale.

Autres co-récepteurs utilisés par VIH-1

En même temps que CGR5, deux groupes identifièrent les récepteurs de chimiokines CCR-3 et CCR2b comme des co-récepteurs potentiels pour VIH-1 [1, 2]. Mais leur activité semblait restreinte à certaines souches virales, et n'était pas mise en évidence par tous les tests. Le rôle des voies d'entrée CCR3 et CCR2b dans le cycle naturel de VIH-1 fut donc considéré au mieux comme secondaire. Si cette vision semble justifiée pour CCR2b, dont l'utilisation comme co-récepteur n'a été montrée, à ma connaissance, que pour une seule souche, le rôle de CCR3 pourrait être plus important. En effet, son absence d'activité, ou sa relative inefficacité par rapport à CCR5 dans certains tests, sont probablement dues à un très faible niveau d'expression après transfection [1]. Quand CCR3 est synthétisé à un niveau suffisant, il semble permettre l'infection des cellules $\mathrm{CD} 4^{+}$par toutes les souches VIH-1 utilisant CCR5, et cela avec une efficacité comparable [3, 4]. Par ailleurs, l'expression du gène CCR3, qu'on pensait limitée aux polynucléaires éosinophiles, a été retrouvée dans les lymphocytes $\mathrm{T}$ infiltrant les tumeurs (TIL) [5], et dans la microglie [3]. La voie d'entrée CCR3 pourrait ainsi jouer un rôle dans l'infection du système nerveux central par VIH-1.

Le même type d'explication technique pourrait expliquer les résultats discordants obtenus pour US28, un récepteur de CC chimiokines codé par le cytomégalovirus humain (CMV). Comme CCR3, US28 est assez mal exprimé après transfert génique [6]. En revanche, en tant que protéine virale, il est probablement abondant dans les cellules infectées par CMV, parmi lesquelles les lymphocytes $\mathrm{CD}^{+}$et les macrophages. Même si la durée de vie des cellules infectées par CMV est limitée, la surexpression d'un co-récepteur d'entrée pourrait en faire des cibles privilégiées pour VIH-1, et ainsi accélérer sa diffusion. On retrouve d'ailleurs assez fréquemment des cellules co-infectées par ces deux virus dans certains tissus (voir discussion dans [6]).

A la liste des co-récepteurs potentiels de VIH-1 se sont récemment ajoutées deux molécules, désignées pour l'instant STRL33/bonzo, et Gpr15/ bob. qui ne sont pas des récepteurs de chimiokines stricto sensu car leurs ligands sont inconnus, mais appartiennent clairement à cette famille $[5,7,8]$. Ces molécules sont produites, entre autres, dans les cellules cibles naturelles des VIH, lymphocytes et macrophages, et semblent être en fait plus efficacement utilisées par VIH-2 et SIV, que par VIH-1 (voir plus bas).

L'utilisation par VIH-1 de co-récepteurs autres que CCR5, in vivo, est indiquée par les cas d'infection à VIH-1, et même de Sida, récemment rapportés chez des sujets $\triangle C C R 5$ [911]. Ces études n'ont pas identifié la ou les voies d'entrée utilisées par VIH-1 comme alternative à CCR5.

\section{Les co-récepteurs utilisés par VIH-2 et SIV}

Le deuxième groupe de lentivirus de primates, est constitué par VIH-2 et 
par les SIV qui infectent le macaque rhésus (SIVmac) et le mangabey (SIVsm). Leur réplication dans certaines lignées cellulaires $\mathrm{CD}^{+}$totalement résistantes à l'infection par VIH-1 [12], suggérait l'existence de co-récepteurs utilisés seulement par VIH-2 et SIV. Ces propriétés ont été récemment observées pour un récepteur orphelin, désigné Gpr1, qui permet l'infection de cellules CD $4^{+}$par SIV, et non par VIH-1 [8]. Son activité vis-à-vis de VIH-2 n'est pas encore connue. Bien que fonctionnels pour certaines souches VIH-1, les co-récepteurs STRL33/bonzo et Gpr15/bob semblent utilisés plus efficacement par VIH-2 et/ou par SIV $[7,8]$. Un niveau d'expression modéré de ces molécules dans certaines lignées pourrait expliquer leur permissivité sélective pour VIH-2 et SIV. Mais des études plus poussées de l'expression de ces nouveaux co-récepteurs sont nécessaires avant de pouvoir affirmer leur rôle.

Nous avons montré que les principaux co-récepteurs de VIH-1, c'est-àdire CCR5, CXCR4, et CCR3, permettaient l'infection de cellules $\mathrm{CD} 4^{+}$ par des souches de VIH-2, primaires ou adaptées aux lignées [4]. Nous avons cependant noté plusieurs différences avec VIH-1, la principale étant une moindre dépendance vis-à-vis de la voie d'entrée CCR5. La plupart des souches VIH-2 primaires sont en effet capables de se répliquer dans des lymphocytes $\triangle C C R 5$ et, pour certaines, par une voie indépendante de CXCR4. Si le défaut d'expression de CCR5 ne confère pas une protection absolue contre l'infection par VIH-1, la protection contre VIH-2 est sans doute minime, ou nulle.

Comme VIH-2, SIVmac et SIVmn peuvent infecter des cellules $\mathrm{CD} 4^{+}$ exprimant CCR5, d'origine humaine ou simienne [13]. En revanche, une différence marquante avec VIH-1 et VIH-2 est l'apparente incapacité des virus simiens d'utiliser CXCR4, et cela même pour les SIV isolés d'animaux au stade Sida.

\section{L'interaction de VIH avec ses co-récepteurs}

S'il est clairement établi que la coexpression de CD4 et de certains récepteurs de chimiokines à la surface cellulaire permet l'infection par VIH, les mécanismes moléculaires mis en jeu sont loin d'être élucidés. La sous-unité de surface (gp120) des glycoprotéines d'enveloppe de VIH gouverne le choix du co-récepteur, en particulier entre CCR5 et CXCR4 pour VIH-1, et semble interagir directement avec ces molécules [1416]. Nous avons montré que la deuxième des trois boucles extracellulaires de CXCR4 était nécessaire à la fonction de co-récepteur, et proposé son interaction avec le domaine variable V3 de gp120 [17, 18]. En revanche, on a pas pu définir précisément les domaines de CCR5 qui sont responsables de l'interaction avec gp120, et ils semblent différer selon les souches [19].

Par ailleurs, le fait qu'une même souche virale puisse utiliser plusieurs co-récepteurs, qui n'ont pratiquement aucun motif conservé dans leurs parties extracellulaires, comme par exemple CCR5, CXCR4, et US28, est difficile à concilier avec une vision stéréo-spécifique de l'interaction gp120/co-récepteur. Il faudrait pour cela imaginer que gp120 possède plusieurs sites de reconnaissances pour les co-récepteurs, ou bien que ceux-ci ont, par coïncidence, une conformation commune qui n'est pas partagée par d'autres récepteurs de chimiokines pourtant proches. Il semble plus simple d'envisager que la fonction de co-récepteur de VIH dépende de la possibilité d'interagir avec gp120, mais aussi d'autres propriétés qui restent à définir. Même dans le cas d'un co-récepteur efficace, comme CCR5, l'interaction avec gp120 semble de faible affinité $[15,16]$. Cette interaction pourrait être assez peu spécifique, et CCR5 facilement remplacé par un autre membre de cette famille de récepteurs, pourvu qu'il soit exprimé à un niveau suffisant, et doué de propriétés nécessaires aux étapes initiales de l'entrée virale. Parmi cellesci, on peut penser que les co-récepteurs doivent être présents à proximité de CD4 (co-localisation, voire interaction directe), et avoir une mobilité latérale suffisante. Il semble en effet nécessaire, après la phase d'adsorption virale, que le complexe CD4/co-récepteur/gp120 soit écarté, afin que la sous-unité transmembranaire (gp41) de l'enveloppe virale puisse accéder à la membrane de la cellule cible, et initier la fusion membranaire $\left(\mathrm{m} / \mathrm{s} \mathrm{n}^{\circ} 2\right.$, vol. 13 , p. 264).

Ces propriétés pourraient être partagées par de nombreux récepteurs de chimiokines, ou récepteurs à 7 domaines transmembranaires apparentés. La liste des co-récepteurs de VIH n'est donc pas close.

M.A.

1. Choe H, Farzan M, Sun Y, Sullivan N, Rollins B, Ponath PD, Wu L, Mackay CR, LaRosa G, Newman W, Gerard N, Gerard C, Sodroski J. The $\beta$ chemokine receptors CCR3 and CCR5 facilitate infection by primary HIV-1 isolates. Cell 1996; 85 : $1135-48$.

2. Doranz BJ, Rucker J, Yi Y, Smyth RJ, Samson M, Peiper SC, Parmentier M, Collman RG, Doms RW. A dual-tropic primary HIV-1 isolate that uses fusin and the $\beta$-chemokine receptors CKR- 5 , CKR3, and CKR-2B as fusion cofactors. Cell $1996 ; 85$ : 1149-58.

3. He J, Chen Y, Farzan M, Choe H, Ohagen A, Gartner S, Busciglio J, Yang X, Hofmann W, Newman W, Mackay CR, Sodroski J, Gabuzda D. CCR3 and CCR 5 are co-receptors for HIV-1 infection of microglia. Nature 1997 ; 385 : 645-9.

4. Sol N, Ferchal F, Braun J, Pleskoff O, Tréboute C, Ansart I, Alizon M. Usage of the coreceptors CCR-5, CCR-3, and CXCR-4 by primary and cell line-adapted human immunodeficiency virus type 2. J Virol $1997 ; 71$ (sous presse).

5. Liao S, Alkhatib G, Liao F, Peden KWC, Sharma G, Berger EA, Farber JM. STRL33, a novel chemokine receptor-like protein, functions as a fusion cofactor for both macrophage-tropic and T cell line-tropic HIV-1. J Exp Med 1997 ; 185 : 2015-23.

6. Pleskoff O, Tréboute C, Brelot A, Heveker N, Seman M, Alizon M. Identification of a chemokine receptor encoded by human cytomegalovirus as a cofactor for HIV-1 entry. Science $1997 ; 276$ : $1874-8$.

7. Deng H, Unutmaz D, KewalRamani VN, Littman DR. Expression cloning of new receptors used by simian and human immunodeficiency viruses. Nature $1997 ; 388: 296-300$.

8. Farzan M, Choe H, Martin K, Marcon L, Hofmann W, Karlsson G, Sun Y, Barrett P, Marchand N, Sullivan N, Gerard N, Gerard C, Sodroski J. Two orphan seven-transmembrane segment receptors which are expressed in CD4-positive cells support simian immunodeficiency virus infection. J Exp Med 1997 ; 186 : 405-11.

9. Biti R, French R, Young J, Bennetts B, Stewart G, Liang T. HIV-1 infection in an individual homozygous for the CCR-5 deletion allele. Nature Med 1997 ; 5 : 252-3.

10. O'Brien T, Winkler C, Dean M, Nelson JAE, Carrington M, Michael NL, White II GC. HIV-1 infection in a man homozygous for CCR-5 $\Delta 32$. Lancet $1997 ; 349: 1219$. 
11. Theodorou I, Meyer L, Magierowska M, Katlama C, Rouzioux C, Group SS. HIV-1 infection in an individual homozygous for CCR-5 $\Delta 32$. Lancet 1997; 349: 1219-20.

12. Clapham PR, Blanc D, Weiss RA. Specific cell surface requirements for the infection of CD4positive cells by human immunodeficiency virus types 1 and 2 and by simian immunodeficiency virus. Virology 1991; 181: 703-15.

13. Chen Z, Zhou P, Ho DD, Landau NR, Marx PA. Genetically divergent strains of simian immunodeficiency virus use CCR5 as a coreceptor for entry. J Virol 1997; 71 : 2705-14.

14. Lapham CK, Ouyang J, Chandrasekhar B, Nguyen NY, Dimitrov DS, Golding H. Evidence for cell-surface association between fusin and the
CD4-gp120 complex in human cell lines. Science $1996 ; 274: 602-5$.

15. Trkola A, Dragic T, Arthos J, Binley JM, Olson WC, Allaway G, Martin SR, Cheng-Mayer C, Robinson J, Maddon PJ, Moore JP. CD4-dependent, antibody-sensitive interactions between HIV-1 and its coreceptor CCR5. Nature 1996; 384: 184-7.

16. Wu L, Gerard NP, Wyatt R, Choe H, Parolin C, Ruffing N, Borsetti A, Cardoso AA, Desjardin E, Newman W, Gerard C, Sodroski J. CD4-induced interaction of primary HIV-1 gp120 glycoproteins with the chemokine receptor CCR-5. Nature 1996 ; 384: 179-83.

17. Brelot A, Heveker N, Pleskoff O, Sol N, Alizon M. Role of the first and third extracellular domains of CXCR-4 in human immunodeficiency virus coreceptor activity. J Virol 1997; 71 : 4744-51. 18. Pleskoff O, Sol N, Labrosse B, Alizon M. Human immunodeficiency virus strains differ in their ability to infect CD4+ cells expressing the rat homolog of CXCR-4 (fusin). J Virol 1997; 71 : 3259-62.

19. Rucker J, Samson M, Doranz BJ, Libert F, Berson JF, Yi Y, Smyth RJ, Collman RG, Broder CC, Vassart G, Doms RW, Parmentier M. Regions in $\beta$ chemokine receptors CCR5 and CCR2b that determine HIV-1 cofactor activity. Cell 1996; 87 : 437-46.

20. Minty A. Une nouvelle famille de cytokines inflammatoires. Med Sci 1991; 7 : 578-88.

\section{BREVES}

L'IKB kinase, la pièce manquante du puzzle. Les grandes lignes de la transmission du signal aboutissant à l'activation du facteur de transcription NF- $\mathrm{kB}$ sont bien connues des lecteurs de médecine/sciences. Un signal inflammatoire, par exemple des cytokines, aboutit à la phosphorylation de la protéine IкB qui est ainsi désignée pour être victime d'une dégradation protéolytique après addition de résidus d'ubiquitine et action des protéasomes $(\mathrm{m} / \mathrm{s} \ldots)$. Le facteur NFкB (sous-unité p55 et p60) est alors libéré, passe dans le noyau où il se fixe à ses sites d'ADN spécifiques. Ce qui manquait à la connaissance de ces phénomènes restait la kinase activée par le signal inflammatowe et capable de phosphoryler IкB. C'est maintenant chose faite: l'équipe de M. Karin (La Jolla, CA, USA) vient de rapporter la purification de l'IкB-kinase et le clonage d'un ADN complémentaire codant pour l'une de ses sous-unités. Celleci a le potentiel de coder pour une sérine/thréonine kinase de 744 acides aminés qui est activée par des cytokines telles que le TNF et
l'IL-1 [1]. Cette nouvelle protéinekinase a été dénommée IKK et la sous-unité dont l'ADNc a été cloné IKK $\alpha$. IKK est inactivée par la protéine phosphatase PP2A, ce qui laisse supposer que son activation passe par une phosphorylation. Reste donc à isoler la kinase IKKK... et les éventuelles autres kinases situées plus en amont. Après tout, il manque encore probablement quelques pièces au puzzle!

[1. DiDonato JA, et al. Nature 1997; 388: 548-54.] 